\title{
FAS $-670 A>G$ promoter polymorphism is associated with soluble Fas levels in primary Sjögren's syndrome
}

\author{
B.A. Treviño-Talavera ${ }^{1}$, C.A. Palafox-Sánchez ${ }^{1,2}$, J.F. Muñoz-Valle ${ }^{1}$, \\ G. Orozco-Barocio ${ }^{2}$, R.E. Navarro-Hernández ${ }^{1}$, M. Vázquez-Del Mercado ${ }^{1}$, \\ I. García de la Torre ${ }^{2}$ and E. Oregon-Romero ${ }^{1}$
}

${ }^{1}$ Department of Molecular Biology and Genon
University Center of Health Sciences, University
Guadalajara, Jalisco, México
${ }^{2}$ Department of Rheumatology, Western General
Zapopan, Jalisco, México
Corresponding author: E. Oregon-Romero
E-mail: oregon_edith@hotmail.com
Genet. Mol. Res. 13 (3): 4831-4838 (2014)
Received May 14, 2013
Accepted October 10, 2013
Published July 2, 2014
DOI http://dx.doi.org/10.4238/2014.July.2.12

ABSTRACT. Primary Sjögren's syndrome (pSS) is a chronic systemic autoimmune disease characterized by lymphocytic infiltration of exocrine glands. Soluble Fas receptor (sFas) has been suggested as a Fas-mediated apoptosis blocker that could impair clonal deletion in infiltrated autoreactive cells. The FAS $-670 \mathrm{~A}>\mathrm{G}$ promoter polymorphism has been studied in pSS. However, a relationship between FAS $-670 \mathrm{~A}>\mathrm{G}$ promoter polymorphism and sFas levels in pSS had not been found. We examined this relationship in 77 Mexican pSS patients and 84 healthy subjects were included. 
Genotypes were identified by PCR-RFLP, and Fas soluble levels were quantified by ELISA. No significant differences between allele and genotype frequencies were found between these two groups. The sFas levels in the serum of pSS patients were significantly higher than in controls (9961 vs $8840 \mathrm{pg} / \mathrm{mL}$, respectively). In addition, AA genotype carriers had significantly higher levels of sFas than GG carriers (pSS: 10,763 and $9422 \mathrm{pg} / \mathrm{mL}$; controls: 9712 and $8305 \mathrm{pg} / \mathrm{mL}$, respectively). An additive model analysis between genotypes ( $\mathrm{AG}+\mathrm{GG}$ vs $\mathrm{AA}$ ) in both groups, demonstrated a significant association between carriers of the A allele and high sFas levels. In conclusion, carrying the double dose of A allele of FAS $-670 A>G$ polymorphism is associated with high levels of sFas in pSS, but it is not a susceptibility marker for pSS.

Key words: Primary Sjögren syndrome; Soluble Fas; FAS gene; FAS $-670 \mathrm{~A}>\mathrm{G}$ polymorphism; Fas receptor

\section{INTRODUCTION}

Primary Sjögren's syndrome (pSS) is a chronic systemic autoimmune disease characterized by lymphocytic infiltration of the exocrine glands, diminished secretory function and several extraglandular manifestations. Autoantibodies to Ro/SSA and La/ SSB are the most frequent autoantibodies in pSS patients. The disease affects 9 times as many middle-aged women as men (Mavragani and Moutsopoulos, 2010).

Apoptosis of glandular epithelial cells has been postulated as an initial event of primary Sjögren's syndrome. High expressions of Fas receptor (CD95) and FasL (CD95L) have been found in minor salivary gland biopsies of pSS patients. In addition, autoantigens expressed on the surface of apoptotic bodies, lead to persistent exposure to antigen-presenting cells (Ohlsson et al., 2002; Bolstad et al., 2003). However, a decrease or blockage of apoptotic mechanisms has also been proposed (Ohlsson et al., 2001). These mechanisms could impair clonal deletion and activation-induced cell death of autoreactive cells, thus causing chronic infiltration of the exocrine glands in pSS patients (Hondowicz et al., 2008).

The soluble form of Fas receptor ( $\mathrm{sFas}$ ), a product of alternatively spliced Fas mRNA, has been suggested as a Fas-mediated apoptosis blocker (Cheng et al., 1994). High levels of sFas have been previously reported in the serum of patients with autoimmune diseases (Ates et al., 2004; Hao et al., 2006; Arasteh et al., 2010). The mechanisms underlying the increase in sFas remain unknown. Polymorphisms in the Fas receptor gene may affect its expression and that of the soluble form (Kanemitsu et al., 2002). The FAS gene is located on 10q24.1 and is highly polymorphic (Niemela et al., 2006). The $-670 A>G(r s 1800682)$ polymorphism is found in the consensus sequence of the gamma interferon activation site, which binds the cytokine response factor STAT1. The A allele leads to enhanced binding STAT1 and therefore is associated with a tendency of higher transcriptional activity than the G allele (Kanemitsu et al., 2002). The AA genotype is associated with the highest sFas levels in comparison to the other genotypes in a healthy 
Tunisian population (Mahfoudh et al., 2007). The FAS -670A allele was associated with susceptibility to systemic lupus erythematosus (SLE) in a Japanese population (Kanemitsu et al., 2002). However, contradictory results between populations have been found in several autoimmune diseases (Kanemitsu et al., 2002; Niino et al., 2002; van Veen et al., 2002; Hiraide et al., 2005; Agarwal et al., 2007; Arasteh et al., 2010). A study in Caucasians found no association of the polymorphism with pSS susceptibility; however, the authors did not assess the possible influence of the FAS $-670 \mathrm{~A}>\mathrm{G}$ polymorphism on sFas levels (Mullighan et al., 2004). The aim of this study was to evaluate the association between FAS $-670 \mathrm{~A}>\mathrm{G}$ and sFas levels in $\mathrm{pSS}$, a relationship that has not previously been addressed.

\section{MATERIAL AND METHODS}

\section{Study population}

Seventy-seven Mexican pSS patients who fulfilled the 2002 American-European classification criteria (Vitali et al., 2002) and 84 unrelated ethnically matched healthy subjects (HS) were included in the study. Patients were recruited from the Rheumatology Department at Hospital General de Occidente in Zapopan, Jalisco, Mexico.

\section{Ethical considerations}

The study was performed in accordance with the ethical standards provided in the Declaration of Helsinki. This study was approved by the Committee of Ethics at Hospital General de Occidente, Zapopan, Jalisco, Mexico (Approval No. 210-10).

\section{Laboratory assessment}

Peripheral blood was obtained from patients and healthy subjects. Blood cell count was obtained using an automatic cell counter (Cell-dyn 1700 Abbott Diagnostics, Lake Forest, IL, USA). Erythrocyte sedimentation rate (ESR) was assayed by the Wintrobe method; serum levels of rheumatoid factor (RF) and C-reactive protein (CRP) were measured by latex turbidimetric immunoassay (A25-Autoanalyzer; Biosystems S.A., Spain). Anti-Ro/SSA and anti-La/SSB autoantibodies were identified separately by indirect ELISA (Bindazyme SSA/ Ro or SSB/La, The Binding Site, UK).

\section{Genotyping}

DNA was isolated from peripheral blood mononuclear cells (PBMCs) according to the Miller method (Miller et al., 1988). The $-670 \mathrm{~A}>\mathrm{G}$ polymorphism in the FAS gene was identified by polymerase chain reaction-restriction fragment length polymorphism (PCR-RFLP) analysis. Briefly, a 193-bp fragment containing the promoter polymorphism was amplified in a thermal cycler (Techne TC-312, UK) using the following primers: 5'-ATAGCTGGGGCTATGCGATT-3' (forward) and 5'-CATTTGACTGGGCTGTCCAT-3' (reverse). Cycling conditions were previously described (Sun et al., 2004). The amplified 
fragments were digested with $4 \mathrm{U}$ ScrFI (New England Biolabs, UK), for $3 \mathrm{~h}$ at $37^{\circ} \mathrm{C}$. The AA genotype is indicated by an uncut 193-bp fragment; fragments of 136 and 57 bp represent the GG genotype. Heterozygosity was indicated by the presence of 193-, 136- and 57-bp fragments. The amplified and digested products were observed on $3 \%$ agarose gels (Sigma-Aldrich, UK) stained with ethidium bromide and visualized with UV light on the Kodak Electrophoresis Documentation and Analysis System (Kodak EDAS 290, USA). The allele distributions for the FAS $-670 A>$ G polymorphism were in HardyWeinberg equilibrium.

\section{Soluble Fas levels}

Serum samples were obtained from patients and healthy subjects and sFas levels were quantified by a sandwich ELISA according to manufacturer protocol (Quantikine Human sFas/TNFRSF6 Immunoassay, R\&D Systems, USA); the sensitivity of the assay was $20 \mathrm{pg}$ / $\mathrm{mL}$. The sample concentrations were calculated using a calibration curve prepared by sequential dilutions of a reconstituted sFas standard.

\section{Statistical analysis}

Allele and genotype frequencies in pSS patients and healthy subjects were compared by the chi-square test $\left(\chi^{2}\right)$. The Student $t$-test and the Mann-Whitney U-test (2-tailed) were used to compare groups; data are reported as means $\pm \mathrm{SD}$. P values $<0.05$ were considered to be statistically significant. Data were analyzed using statistical software packages (Epi-Info 3.5.1 version for Windows, CDC, and SPSS Statics 17.0 version for Windows, USA).

\section{RESULTS}

The genotype frequencies in pSS were 26 (AA), 41.5 (AG), and 32.5\% (GG), and 23.8 (AA), 50 (AG), and 26.2\% (GG) in HS. Heterozygotes were the most frequent in both groups. There were no significant differences in allele and genotype frequencies $(\mathrm{P}=0.0538$ and $\mathrm{P}=$ 0.712 , respectively).

sFas levels were higher in pSS patients than in HS (9961 vs $8940 \mathrm{pg} / \mathrm{mL}, \mathrm{P}=0.006$ ) (Figure 1A). In order to investigate the association of FAS $-670 \mathrm{~A}>\mathrm{G}$ with soluble protein, $\mathrm{sFas}$ levels were compared across genotypes in both groups. The AA genotype was associated with higher levels of sFas than the GG genotype in pSS $(10,763$ vs $9422 \mathrm{pg} / \mathrm{mL}, \mathrm{P}=0.026)$ and HS (9712 vs $8305 \mathrm{pg} / \mathrm{mL}, \mathrm{P}=0.045$ ) (Figure 1B).

Additive model analyses were performed to determine the allele influence on sFas levels. In both groups, carriers of the A allele exhibited higher levels of sFas than non-carriers (pSS: 10,226 vs $9422 \mathrm{pg} / \mathrm{mL}$; HS: 9187 vs $8305 \mathrm{pg} / \mathrm{mL}$ ), although the differences were not statistically significant. In addition, pSS patients with the AA genotype had higher levels of sFas in comparison to $\mathrm{G}$ allele carriers in the pSS $(10,763$ vs $9679 \mathrm{pg} / \mathrm{mL}, \mathrm{P}=0.045)$ and HS groups (9712 vs $8652 \mathrm{pg} / \mathrm{mL}$ ) (Figure 2). 


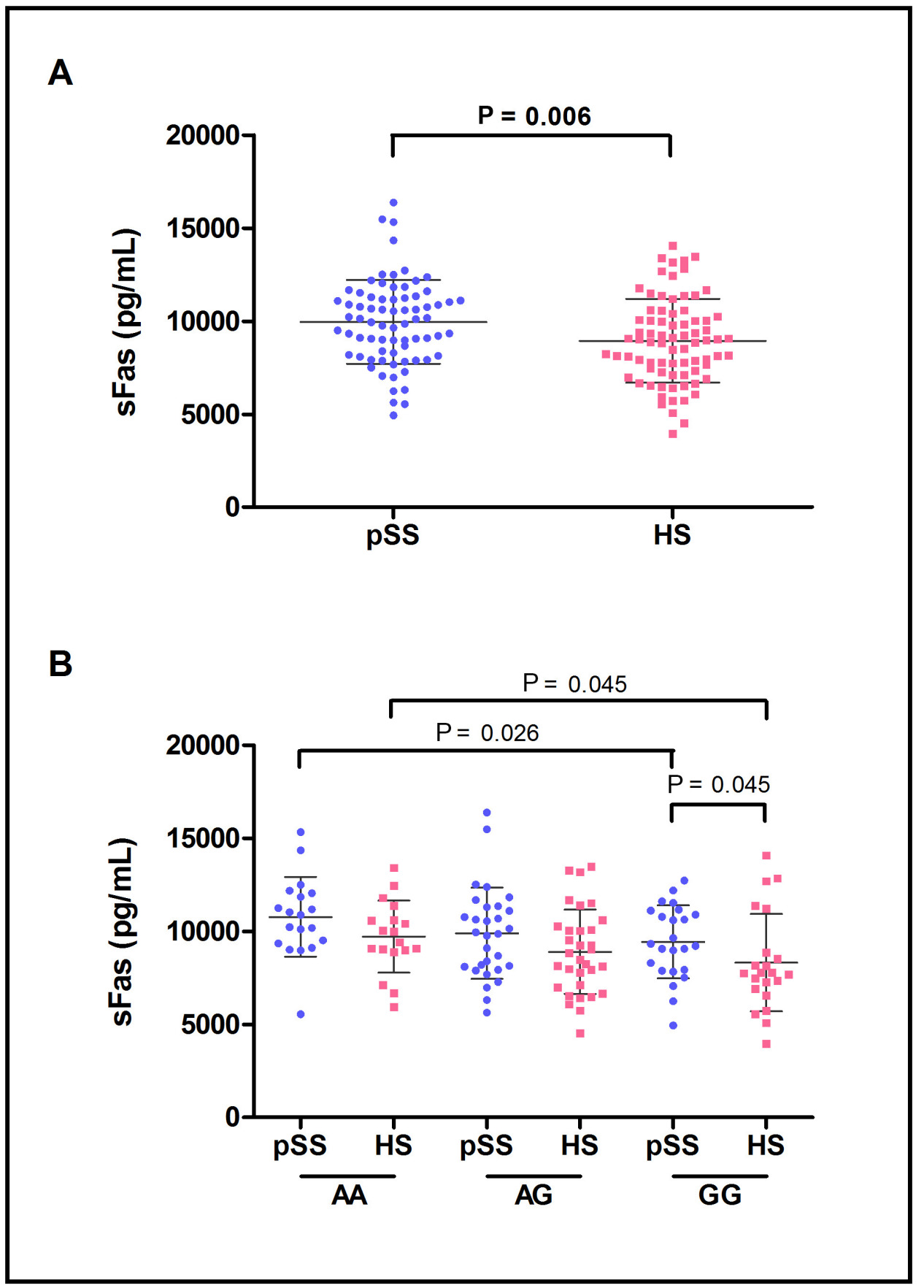

Figure 1. Soluble Fas levels in primary Sjögren syndrome patients and healthy subjects. A. By group. B. By FAS $-670 \mathrm{~A}>\mathrm{G}$ genotype. Data are shown as means $\pm \mathrm{SD}$. HS = healthy subjects; $\mathrm{pSS}=$ primary Sjögren's syndrome; sFas $=$ soluble Fas. 


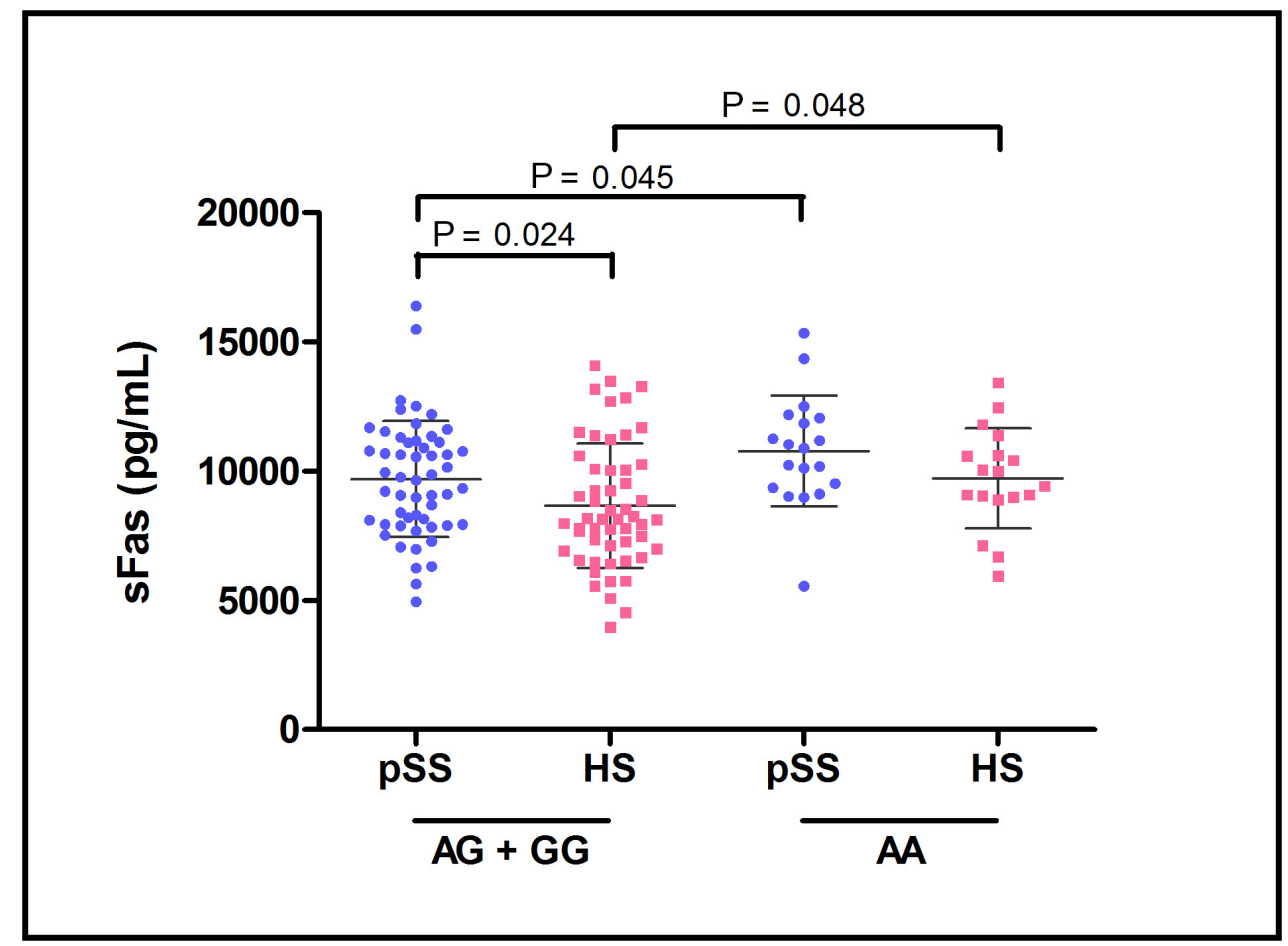

Figure 2. Soluble Fas levels in primary Sjögren syndrome patients and healthy subjects, according to the additive model. Data are shown as means \pm SD. HS = healthy subjects; $p S S=$ primary Sjögren's syndrome; $s F a s=$ soluble Fas.

\section{DISCUSSION}

Apoptosis is a regulated cell death that occurs normally in multicellular organisms. The Fas-Fas ligand pathway is the most important mechanism of extrinsic apoptosis. Through this process, autoreactive cells are eliminated to maintain self-tolerance. However, aberrations in apoptosis lead to development and maintenance of autoimmune processes. It has been proposed that polymorphisms in the promoter region of the FAS gene modulate receptor expression and thus apoptosis in autoimmune and non-autoimmune diseases.

The FAS $-670 \mathrm{~A}>\mathrm{G}$ polymorphism has been linked to susceptibility to autoimmune diseases such as SLE and multiple sclerosis (Kanemitsu et al., 2002; van Veen et al., 2002; Agarwal et al., 2007). However, another group of studies in different populations found no such association (Niino et al., 2002; Arasteh et al., 2010). The difference in these association studies may be due to differences in genotype distributions between populations. A previous study of the FAS $-670 \mathrm{~A}>\mathrm{G}$ polymorphism found no differences in allele or genotype frequencies between pSS patients and HS in a Caucasian population (Mullighan et al., 2004). We also found no association between the polymorphism and pSS susceptibility in our tested population. Although genetic background is one of the predisposing factors for the development of autoimmunity, the FAS $-670 \mathrm{~A}>\mathrm{G}$ polymorphism likely has no significant effect on susceptibil- 
ity to pSS. However, it has been reported the $-670 \mathrm{~A}>\mathrm{G}$ polymorphism is in linkage disequilibrium with other variations in the FAS gene (Hiraide et al., 2005). Therefore, further studies are needed to determine whether susceptibility to pSS could be due to a specific haplotype.

High levels of sFas have been reported in autoimmune diseases such as SLE, rheumatoid arthritis, and systemic sclerosis (Ates et al., 2004; Hao et al., 2006; Arasteh et al., 2010). The role of sFas has not been clarified, but it may inhibit Fas-mediated apoptosis by competing with the receptor for FasL binding (Cheng et al., 1994). An increase of sFas could lead to apoptotic resistance in infiltrating lymphocytes in the salivary glands of pSS patients. Consistent with this hypothesis, we found high levels of sFas in pSS patients in comparison to HS.

Whether elevated levels of $\mathrm{sFas}$ are a cause or a reflection of autoimmune disease remains unknown. The A allele of the $-670 \mathrm{~A}>\mathrm{G}$ polymorphism provides high binding activity for the STAT1 transcription factor (Kanemitsu et al., 2002). The A allele could increase FAS gene expression and elicit an increase in sFas levels. Consistent with this, we found high sFas levels in AA genotype carriers of both groups (pSS: $\mathrm{P}=0.026$; HS: $\mathrm{P}=0.045$, vs $\mathrm{GG}$ genotype carriers). In addition, heterozygous carriers tended to express higher sFas levels than GG carriers. Our results are similar to those reported in a healthy Tunisian population in which sFas levels increased in an A allele dose-dependent manner (Mahfoudh et al., 2007).

Although the additive model analysis showed that A allele carriers tend to exhibit higher levels of sFas, this increase is not statistically significant. When the additive model for the $\mathrm{G}$ allele was performed, we found higher levels of sFas in AA genotype carriers than in $\mathrm{G}$ allele carriers. Our results suggest that the A allele of FAS $-670 A>G$ increases transcription, but only increases sFas levels in A homozygotes.

Fas-induced apoptosis may be blocked in pSS, which would explain the prolonged survival of infiltrating lymphocytes in the salivary glands (Ohlsson et al., 2001). High levels of sFas due to allele homozygosity could block apoptosis of autoreactive lymphocytes and lead to their accumulation. It is possible that increased pSS severity is associated with sFas levels. However, further studies are needed to confirm this hypothesis, since it is unknown whether the FAS $-670 \mathrm{~A}>\mathrm{G}$ polymorphism modulates membrane expression of the Fas receptor.

In conclusion, our findings suggest that a double dose of the FAS -670 A allele is associated with high levels of sFas in pSS but is not a susceptibility marker in pSS.

\section{Conflicts of interest}

The authors declare no conflict of interest.

\section{ACKNOWLEDGMENTS}

Research supported by Grant \#88046 to E. Oregon-Romero of the CONACYT (Fondo Sectorial SSA/IMSS/ISSSTE-CONACYT, México-Universidad de Guadalajara).

\section{REFERENCES}

\footnotetext{
Agarwal K, Czaja AJ and Donaldson PT (2007). A functional Fas promoter polymorphism is associated with a severe phenotype in type 1 autoimmune hepatitis characterized by early development of cirrhosis. Tissue Antigens 69: 227-235. Arasteh JM, Sarvestani EK, Aflaki E and Amirghofran Z (2010). Fas gene polymorphisms in systemic lupus erythematosus and serum levels of some apoptosis-related molecules. Immunol. Invest. 39: 27-38.
} 
Ates A, Kinikli G, Turgay M and Duman M (2004). The levels of serum-soluble Fas in patients with rheumatoid arthritis and systemic sclerosis. Clin. Rheumatol. 23: 421-425.

Bolstad AI, Eiken HG, Rosenlund B, Alarcon-Riquelme ME, et al. (2003). Increased salivary gland tissue expression of Fas, Fas ligand, cytotoxic T lymphocyte-associated antigen 4, and programmed cell death 1 in primary Sjögren's syndrome. Arthritis Rheum. 48: 174-185.

Cheng J, Zhou T, Liu C, Shapiro JP, et al. (1994). Protection from Fas-mediated apoptosis by a soluble form of the Fas molecule. Science 263: 1759-1762.

Hao JH, Ye DQ, Zhang GQ, Liu HH, et al. (2006). Elevated levels of serum soluble Fas are associated with organ and tissue damage in systemic lupus erythematosus among Chinese. Arch. Dermatol. Res. 297: 329-332.

Hiraide A, Imazeki F, Yokosuka O, Kanda T, et al. (2005). Fas polymorphisms influence susceptibility to autoimmune hepatitis. Am. J. Gastroenterol. 100: 1322-1329.

Hondowicz BD, Fields ML, Nish SA, Larkin J, et al. (2008). Autoantibody production in lpr/lpr gld/gld mice reflects accumulation of CD4+ effector cells that are resistant to regulatory T cell activity. J. Autoimmun. 31: 98-109.

Kanemitsu S, Ihara K, Saifddin A, Otsuka T, et al. (2002). A functional polymorphism in fas (CD95/APO-1) gene promoter associated with systemic lupus erythematosus. J. Rheumatol. 29: 1183-1188.

Mahfoudh W, Bel H Jr, Romdhane A and Chouchane L (2007). A polymorphism in FAS gene promoter correlated with circulating soluble FAS levels. Int. J. Immunogenet. 34: 209-212.

Mavragani CP and Moutsopoulos HM (2010). The geoepidemiology of Sjögren's syndrome. Autoimmun. Rev. 9: A305-A310.

Miller SA, Dykes DD and Polesky HF (1988). A simple salting out procedure for extracting DNA from human nucleated cells. Nucleic Acids Res. 16: 1215.

Mullighan CG, Heatley S, Lester S, Rischmueller M, et al. (2004). Fas gene promoter polymorphisms in primary Sjögren's syndrome. Ann. Rheum. Dis. 63: 98-101.

Niemela JE, Hsu AP, Fleisher TA and Puck JM (2006). Single nucleotide polymorphisms in the apoptosis receptor gene TNFRSF6. Mol. Cell Probes 20: 21-26.

Niino M, Kikuchi S, Fukazawa T, Miyagishi R, et al. (2002). An examination of the Apo-1/Fas promoter Mva I polymorphism in Japanese patients with multiple sclerosis. BMC Neurol. 2: 8.

Ohlsson M, Skarstein K, Bolstad AI, Johannessen AC, et al. (2001). Fas-induced apoptosis is a rare event in Sjögren's syndrome. Lab. Invest. 81: 95-105.

Ohlsson M, Jonsson R and Brokstad KA (2002). Subcellular redistribution and surface exposure of the Ro52, Ro60 and La48 autoantigens during apoptosis in human ductal epithelial cells: a possible mechanism in the pathogenesis of Sjögren's syndrome. Scand. J. Immunol. 56: 456-469.

Sun T, Miao X, Zhang X, Tan W, et al. (2004). Polymorphisms of death pathway genes FAS and FASL in esophageal squamous-cell carcinoma. J. Natl. Cancer Inst. 96: 1030-1036.

van Veen T, Kalkers NF, Crusius JB, van Winsen L, et al. (2002). The FAS-670 polymorphism influences susceptibility to multiple sclerosis. J. Neuroimmunol. 128: 95-100.

Vitali C, Bombardieri S, Jonsson R, Moutsopoulos HM, et al. (2002). Classification criteria for Sjögren's syndrome: a revised version of the European criteria proposed by the American-European Consensus Group. Ann. Rheum. Dis. 61: 554-558. 